\title{
Lipoprotein lipase locus and progression of atherosclerosis in coronary-artery bypass grafts
}

Kent D. Taylor, $\mathrm{PhD}^{1,{ }^{*}}$ Maren T. Scheuner, $\mathrm{MD}, \mathrm{MPH}^{1,{ }^{*}}$ Huiying Yang, $\mathrm{MD}, \mathrm{Ph} \mathrm{D}^{1}$, Yaping Wang, $\mathrm{MD}^{1}$, Talin Haritunians ${ }^{1}$, Nathan Fischel-Ghodsian, $M D^{1}$, Prediman K. Shah, $\mathrm{MD}^{2}$, James S. Forrester, $\mathrm{MD}^{2}$, Genell Knatterud, $P h D^{3}$, and Jerome I. Rotter, $M D^{1}$

\begin{abstract}
Purpose: Our aim was to test whether polymorphisms in the lipoprotein lipase $(L P L)$ gene were associated with the progression of atherosclerosis in grafts examined in the Post-Coronary Artery Bypass Graft Trial (Post-CABG Trial). Methods: 843 subjects in the post-CABG trial were genotyped for the LPL-D9N, N291S, Pvull, (TTTA) , and HindIII polymorphisms. Associations between genotype and angiographically measured progression of atherosclerosis in grafts, medical history, and family history were examined. Results: Greater progression of atherosclerosis was observed in subjects with $L P L$-HindIII $2 / 2$ (56\% versus $42 \%$ of those with other $L P L$ Hindlll genotypes, $P=0.025$ ) and with $L P L(T T T A)_{n} 4 / 4$ (63\% versus $43 \%$ of those with other $(\text { TTTA })_{n}$ genotypes, $\left.P=0.020\right)$. Mantel-Haenszel analysis yielded an odds ratio of 1.84 for the effect of $L P L$ HindllI 2/2 genotype on the progression of atherosclerosis in grafts $(P=0.015)$ and demonstrated that the effect of genotype on progression was of the same magnitude as, but independent of, the effect of drug treatment. Conclusion: The LPL-HindllI 2/2 genotype is a marker for genetic variation in the 3 '-end of $L P L$ that acts as an independent risk factor for the progression of atherosclerosis in grafts examined in the Post-CABG Trial. Genet Med 2004:6(6):481-486.
\end{abstract}

Key Words: coronary artery bypass grafts, lipoprotein lipase, lovastatin, atherosclerosis, coronary arteriosclerosis

In the Post-Coronary Artery Bypass Graft (Post-CABG) Trial, subjects were randomly assigned to receive lovastatin therapy to lower LDL-cholesterol with a goal range of 60 to $85 \mathrm{mg} / \mathrm{dL}$ (aggressive treatment group) or 130 to $140 \mathrm{mg} / \mathrm{dL}$ (moderate treatment group). Coronary angiograms of 1351 subjects at baseline and at an average of 4.3 years later were compared using a quantitative assessment of the severity of graft stenosis. The results of this study demonstrated that aggressive lowering of LDL-cholesterol levels using lovastatin reduced the risk of progression of atherosclerosis in grafts. $^{1}$

However, aggressive lovastatin treatment did not eliminate the progression of atherosclerosis in grafts, suggesting that there are other determinants of this progression. Of these, genetic factors may contribute to one or more steps of the atherosclerosis path: alterations in lipid metabolism, chronic inflammatory condition, plaque rupture, and thrombosis of an acute event. $^{2}$

From the ${ }^{1}$ Medical Genetics Institute, and Division of Cardiology, ${ }^{2}$ Department of Medicine, Cedars-Sinai Medical Center, Los Angeles, California; ${ }^{3}$ Maryland Medical Research Institute, Baltimore, Maryland.

${ }^{*}$ These authors contributed equally to this work.

Kent D. Taylor, PhD, Medical Genetics Institute, SSB-378, Cedars-Sinai Medical Center, 8700 Beverly Blvd., Los Angeles, CA 90048.

Received: May 28, 2004.

Accepted: July 20, 2004.

DOI: 10.1097/01.GIM.0000144012.18935.48
The lipoprotein lipase gene (LPL; triacylglycerol acylhydrolase; EC 3.1.1.34) has been associated with both the atherogenic lipid profile and the progression of atherosclerosis. Premature atherosclerosis can occur in patients with lipoprotein deficiency despite low LDL-cholesterol levels. ${ }^{3}$ The LPL-D9N polymorphism has been associated with the atherogenic lipid profile, ${ }^{4-7}$ coronary atherosclerosis, ${ }^{4}$ and attenuation of LDL lowering by pravastatin. ${ }^{8}$ The $L P L-\mathrm{N} 291 \mathrm{~S}$ polymorphism has also been associated with the atherogenic lipid profile, ${ }^{9-10}$ delayed postprandial triglyceride clearance, ${ }^{11-13}$ increased coronary stenosis in women with ischemic heart disease, ${ }^{14}$ and a more severe dyslipidemia in insulin-resistant subjects. ${ }^{15} \mathrm{LPL}$ $P v u I I$ has been associated with angiographically measured coronary obstruction. ${ }^{16}$ LPL-HindIII has been associated with angiographic severity of coronary artery disease, ${ }^{17-20}$ and with features of insulin resistance. ${ }^{21}$

$L P L$ sequence from 3 ethnic groups revealed that a "recombination hotspot" around exon 6 breaks LPL into two sections: the 5 '-section includes $L P L$-D9N and N291S, and the 3'-section includes LPL-HindIII.22-25 Linkage disequilibrium is high within each section but low between the sections.

The purpose of this study was to test whether these 5 wellcharacterized polymorphisms in LPL were associated with progression of atherosclerosis in saphenous vein coronary artery bypass grafts, and whether these polymorphisms interacted with lipid-lowering therapy to affect the rate of progression. The results identify a genetic determinant of atherosclerosis progression in the 3 '-section of $L P L$. 


\section{METHODS}

\section{Study design}

This genetic association study is ancillary to the Post-Coronary Artery Bypass Graft Trial (Post-CABG Trial). ${ }^{1}$ The associations between the progression of atherosclerosis, measured quantitatively by angiography at baseline and 4 to 5 years later, and genotypes for 5 LPL polymorphisms were determined. All procedures were approved by the institutional review boards of Cedars-Sinai Medical Center and the other centers participating in the Post-CABG Trial. Consent for the clinical trial was obtained prior to enrollment and consent for this genetic study was obtained during follow-up.

\section{Subjects}

A total of 1351 subjects from seven clinical centers throughout North America were included in the clinical trial ${ }^{1}$ and all were eligible as participants in this genetic ancillary study. Inclusion criteria for the clinical trial were as follows: bypass surgery before the study; an LDL-cholesterol level of 130 to 175 $\mathrm{mg} / \mathrm{dL}$; at least one patent vein graft as determined by angiography. Exclusion criteria in the clinical trial were as follows: likelihood of revascularization or death within the study period of 5 years; unstable angina or myocardial infarction within six months before the start of the trial; severe angina; heart failure; and contraindications to the study medications. ${ }^{1}$ Subjects were randomly assigned for treatment to lower LDL-cholesterol levels with lovastatin and cholestyramine, aggressively (target LDL 60-85 mg/dL) or moderately (target LDL 130$140 \mathrm{mg} / \mathrm{dL})$.

\section{Data collection}

All demographic, family history, medical history, and clinical data were collected as part of the Post-CABG Trial. ${ }^{1}$ In brief, the progression of atherosclerosis was quantitatively determined by comparison of an initial angiogram at enrollment with a follow-up angiogram repeated an average of 4.3 years later. Baseline and follow-up angiography were obtained with catheterization techniques that permitted computer-assisted quantitative measurement. An initially patent graft was defined as having progression of atherosclerosis if there was a decrease of $0.6 \mathrm{~mm}$ or more in lumen diameter at the site of greatest change at follow-up. The primary endpoint of the initial study was the percentage of grafts showing progression of atherosclerosis in each subject. "Subjects with progression of atherosclerosis" were defined as those subjects with one or more grafts showing progression.

Complete angiographic data on follow-up and DNA were available for 843 subjects in the genetic study. Of these, cell lines from 192 subjects in the LA cohort were established by transformation of peripheral blood lymphocytes with EpsteinBarr virus (EBV) $26-29$ during years 2 to 3 of the clinical trial. During years 4 to 5 , whole blood was collected from an additional 651 subjects from the other centers. Genetic material was not available from the remaining subjects because the study was started mid-way during the national clinical study. DNA was isolated following standard protocols. ${ }^{30}$

\section{Genotyping}

Conventional agarose gel and fluorescent semiautomated technology were used to genotype the subjects for five different $L P L$ polymorphisms (methods in citations in the legend to Fig. 1).

a)

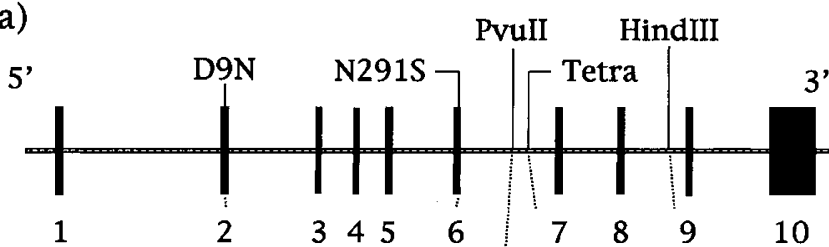

b)
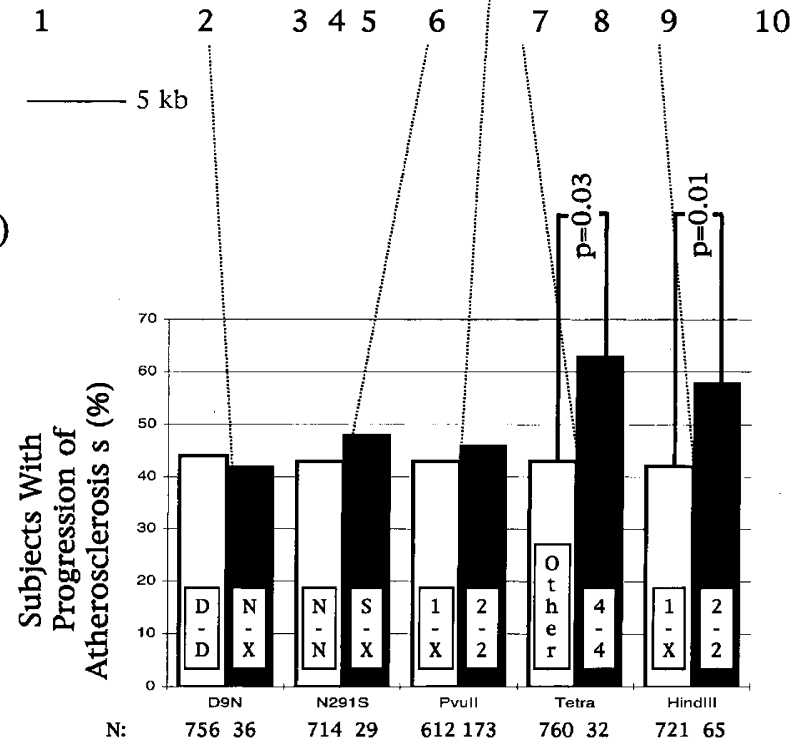

c)

D9N N291S PvuII Tetra HindIII

$\begin{array}{cccccc}\text { Odds Ratio: } & 1.1 & 0.8 & 1.1 & 2.2 & 1.9 \\ \text { (95\% CI) } & 0.6- & 0.4- & 0.8- & 1.1- & 1.2- \\ & 2.6 & 1.7 & 1.6 & 4.6 & 3.2\end{array}$

Fig. 1. Atherosclerosis progression by different $L P L$ markers. a, Location of markers. Location of polymorphisms in the $L P L$ gene was assembled from Genbank accession nos. G187209, G34390, M76722, and M76723. Vertical bars represent exons. "Recombinational hotspot" spans the 3'-half of exon 6 and the 5 '-half of intron 6, ending approximately at the position of $L P L-(\text { TTTA })_{n} \cdot{ }^{24}$ b, Percent subjects with progression of atherosclerosis. Each pair of vertical bars represents two genotype groups for each marker as shown, with the size of each group (N). LPL-D9N and N291S were binned as shown because the low frequency of the rare allele did not permit testing for association. $P v u \mathrm{II}$ and HindIII were binned as shown because of previous reports that the effect of these polymorphisms on atherosclerosis is greatest for the $2 / 2$ homozygote. ${ }^{16,18}$ There was no association observed when the LPL-PvuII polymorphism was tested using other models and hence alternative binning schemes. $L P L-(\text { TTTA })_{n}$ was binned as shown because the $4 / 4$ haplotype showed the greatest association with the progression of atherosclerosis in grafts. Complete progression and genotyping data for each marker were available as listed. Genotypes were determined using published methods modified for semiautomated gel electrophoresis (ABI 373, Genescan \& Genotyper software, Applied Biosystems, Foster City, CA): D9N, ${ }^{4}$ N291S, ${ }^{9}$ PvuII ("1" = site present, "2" = site absent), ${ }^{31}$ (TTTA) (allele 1 is $119 \mathrm{bp}, 2=123 \mathrm{bp}, 3=127 \mathrm{bp}, 4=131 \mathrm{bp}, 5=135 \mathrm{bp}$ ), ${ }^{32}$ HindIII (intron 8; " 1 " = site present, " 2 " = site absent). ${ }^{33}$ " $\mathrm{X}$ " denotes "other" genotypes. Allele frequencies were in Hardy-Weinberg equilibrium for each marker. Percent of subjects with atherosclerosis progression is defined in Methods. c, Odds Ratios for Progression. ORs for progression of atherosclerosis in grafts post-CABG with $95 \% \mathrm{CI}$ are given for each polymorphism. 


\section{Statistical methods}

Statistical analysis was performed using SAS computer software. ${ }^{34}$ All quantitative variables were tested for normality. Distribution of triglycerides and HDL cholesterol levels become near normal with a natural log transformation. The duration of time since $C A B G$ at baseline could not be normalized by various transformations. Therefore, both parametric and nonparametric statistical tests were used in order to test the mean difference between two groups. Differences in proportions were evaluated using the Chi-square method. MantelHaenszel statistics were used for stratified analyses.

Although our primary goal was to evaluate the relationship between the LPL genotype and the progression of atherosclerosis in grafts, we used the following strategy to evaluate the effect of potential confounding variables. First, univariate analyses were conducted to identify variables associated with both the progression of atherosclerosis in grafts and with genotype. Then, interactions between variables were tested using logistic regression analysis with the interaction term as one of the independent variables. Last, the effects (odds ratio) of genotype on progression were compared between models with or without that particular variable.

\section{RESULTS}

\section{Baseline characteristics of subjects in genetic study}

The baseline characteristics of the 843 subjects included in the genetic study and the 508 subjects for whom data were unavailable were compared (Table 1). The study group had a significantly lower frequency of smoking, of prior myocardial infarction, mean steady-state LDL, number of years from CABG to enrollment, and composite clinical events (deaths, nonfatal MI, stroke, PTCA, and CABG). The study group had a significantly higher frequency of aspirin use and mean steady-state HDL-cholesterol. These results suggested that subjects with lesser risk for atherosclerosis progression were disproportionately included in the genetic study.

Within the group of subjects included in the genetic study, there were no significant differences between the aggressive and moderate treatment groups for the same characteristics listed in Table 1 (data not shown).

\section{LPL genotypes and progression of atherosclerosis in grafts}

Fig. 1 diagrams the location of the 5 LPL polymorphisms studied (a), relates LPL genotypes to the percent of subjects showing progression of atherosclerosis in saphenous vein grafts (b), and gives the odds ratios for progression with respect to genotype (c). There was no association between the percentage of subjects exhibiting the progression of atherosclerosis and the polymorphisms in the 5 '-section of $L P L$, e.g., the D9N, N291S, or the PvuII polymorphisms.

A significant difference was observed for polymorphisms in the 3'-section of LPL. For the LPL-HindIII polymorphism, $56 \%$ of subjects with the LPL-HindIII 2/2 genotype exhibited the progression of atherosclerosis compared with $43 \%$ of those
Table 1

Characteristics of subjects in genetic study

\begin{tabular}{|c|c|c|}
\hline Characteristic & $\begin{array}{l}\text { Included } \\
(N=843)\end{array}$ & $\begin{array}{l}\text { Not included } \\
(N=508)\end{array}$ \\
\hline$\overline{\text { Age }(\mathrm{yr}, \text { mean } \pm \mathrm{SE})}$ & $61.6 \pm 0.3$ & $61.5 \pm 0.3$ \\
\hline Caucasian $(\%)$ & 94 & 95 \\
\hline Male (\%) & 92 & 93 \\
\hline Body mass index $\left(\mathrm{kg} / \mathrm{m}^{2}\right.$, mean $\left.\pm \mathrm{SE}\right)$ & $27.7 \pm 0.1$ & $27.9 \pm 0.2$ \\
\hline Current smoking (\%) & 9 & $14^{a}$ \\
\hline History of myocardial infarction & 45 & $55^{c}$ \\
\hline $\begin{array}{l}\text { Time between CABG and enrollment (yr, } \\
\text { mean } \pm \text { SE) }\end{array}$ & $4.7 \pm 0.1$ & $5.1 \pm 0.1^{b}$ \\
\hline Ejection fraction (\%) & $57 \pm 0.4$ & $56 \pm 0.6$ \\
\hline Family history of coronary artery disease & 70 & 70 \\
\hline \multicolumn{3}{|l|}{ Current medications (\%) } \\
\hline Aspirin & 79 & $71^{c}$ \\
\hline Beta-blocker & 24 & 26 \\
\hline Calcium-channel blocker & 24 & 23 \\
\hline Insulin or oral antidiabetic agent & 8 & 10 \\
\hline Thiazide diuretic & 10 & 11 \\
\hline Systolic blood pressure (mm Hg, mean $\pm \mathrm{SE}$ ) & $134.1 \pm 0.6$ & $134.6 \pm 0.8$ \\
\hline Diastolic blood pressure $(\mathrm{mm} \mathrm{Hg}$, mean $\pm \mathrm{SE}$ ) & $79.8 \pm 0.3$ & $79.7 \pm 0.4$ \\
\hline \multicolumn{3}{|l|}{ Baseline lipid levels $(\mathrm{mg} / \mathrm{dl})^{d}$} \\
\hline Total cholesterol & $227.0 \pm 0.9$ & $226.4 \pm 1.1$ \\
\hline LDL cholesterol & $155.4 \pm 0.7$ & $155.6 \pm 0.9$ \\
\hline HDL cholesterol & $39.4 \pm 0.3$ & $39.0 \pm 0.4$ \\
\hline Triglycerides & $160.4 \pm 2.4$ & $159.0 \pm 3.1$ \\
\hline \multicolumn{3}{|l|}{ Steady state lipid levels (mg/dL) } \\
\hline Total cholesterol & $191.1 \pm 1.3$ & $193.9 \pm 1.7$ \\
\hline LDL cholesterol & $114.9 \pm 1.6$ & $119.5 \pm 1.5^{a}$ \\
\hline HDL cholesterol & $44.1 \pm 0.4$ & $42.4 \pm 0.5^{a}$ \\
\hline Triglycerides & $162.6 \pm 3.3$ & $161.7 \pm 4.0$ \\
\hline \multicolumn{3}{|l|}{ Events $(\%)^{e}$} \\
\hline Death & 0 & $3.2^{c}$ \\
\hline MI & 4.4 & 7.5 \\
\hline PTCA & 5.9 & 4.7 \\
\hline CABG & 3.4 & 5.5 \\
\hline Stroke & 1.5 & $4.1^{a}$ \\
\hline Composite $^{f}$ & 11.3 & $22.2^{c}$ \\
\hline
\end{tabular}

Groups included or excluded in this genetic study were compared by ANOVA. Blank $P$ values were nonsignificant at the 0.05 level. ${ }^{a} 0.01 \leq P \leq 0.05 ;{ }^{b} 0.001 \leq$ $P \leq 0.01 ;{ }^{c} P \leq 0.001$.

${ }^{d}$ Values listed are those measured before enrollment. To convert cholesterol values to $\mathrm{mmol} / \mathrm{L}$, multiply by 0.02586 ; to convert triglyceride values to $\mathrm{mmol} / \mathrm{L}$, multiply by 0.01129 .

${ }^{e}$ All events that occurred before the date of follow-up angiography are included. For patients who did not have follow-up angiography, all events that occurred before the follow-up examination completed in the period for scheduled angiography are counted. Events were classified by physicians at each center.

${ }^{f}$ The composite end point was death from CV or unknown causes, nonfatal MI, stroke, PTCA, or CABG.

with either $1 / 1$ or $1 / 2(\mathrm{OR}=1.74,95 \%$ CI $1.07-2.83, P=$ 0.025; chi-square test). Furthermore, the mean proportion of grafts per subject showing a progression of atherosclerosis was 
also significantly increased for those with the LPL-HindIII $2 / 2$ genotype: $39 \%$ for HindIII $2 / 2$ compared with $27 \%$ for $L P L$ HindIII $1 / 1$ and $1 / 2$ combined $(P=0.011$; nonparametric Wilcoxon test). The frequency of the LPL-HindIII 2 allele in this sample was $28 \%$ and in Hardy-Weinberg equilibrium.

The $L P L-(\text { TTTA })_{n} 4 / 4$ genotype was also associated with progression: $63 \%$ of subjects with $L P L-(\mathrm{TTTA})_{n} 4 / 4$ showed progression compared with $43 \%$ for other $L P L$-(TTTA) $)_{n}$ genotypes $(\mathrm{OR}=2.2,95 \% \mathrm{CI} 1.1-4.6 ; P=0.027)$. The frequency of the $L P L-(\text { TTTA })_{n} 4$ allele in this sample was $22 \%$ and in Hardy Weinberg equilibrium. The LPL-HindIII 2 and LPL$(\text { TTTA })_{\mathrm{n}} 4$ alleles were in strong linkage disequilibrium $(P<$ 0.001, data not shown). LPL-HindIII $2 / 2$ and (TTTA) $4 / 4$ combined was also associated with the progression of atherosclerosis at a significance level similar to that of either genotype alone.

Using these same methods, these associations were tested for each study center separately in order to see whether the genetic effect was present in one or two centers only. The percent with progression of atherosclerosis was always higher in subjects with the LPL-HindIII 2/2 genotype at each center, but did not reach statistical significance until the data were combined across the 7 centers.

Since the LPL-HindIII 2/2-(TTTA) $)_{n} 4 / 4$ combination did not identify a more specific haplotype associated with the progression of atherosclerosis in grafts than HindIII 2/2 alone, only the LPL-HindIII polymorphism was studied in the following analyses.

\section{HindIII 2/2 genotype and drug treatment}

The percentages of subjects with atherosclerosis progression when stratified by drug treatment group and LPL-HindIII genotype are shown in Figure 2. The highest percentage of sub-

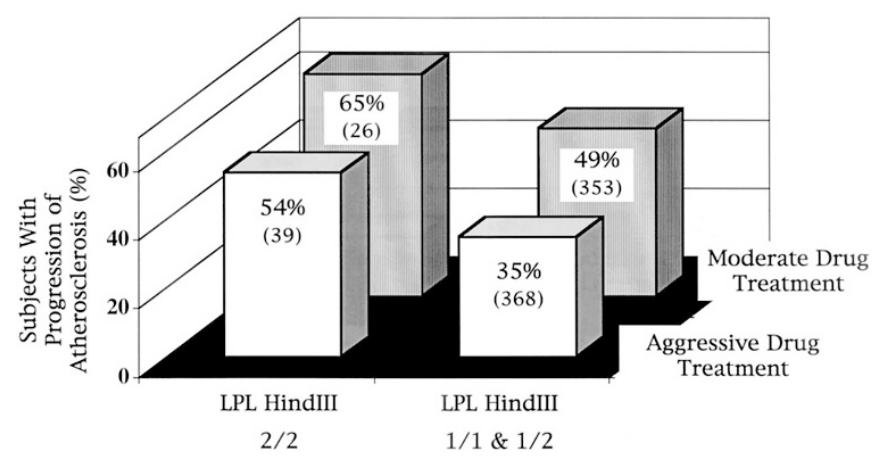

Fig. 2. Progression of atherosclerosis in grafts by $L P L$-HindIII genotype and lovastatin groups. Percent of subjects with progression in grafts, genotype groups, and treatment groups are defined in Methods. Total number of subjects in each group is given on each vertical bar. Within the LPL-HindIII $1 / 1$ and $1 / 2$ genotype group, the moderate drug treatment group had a significantly higher percent of subjects with progression than the aggressive treatment group, $48.7 \%$ compared with $36.6 \%$, OR $=1.65,95 \% \mathrm{CI}=1.24-$ $2.19, P=0.001$. A significant effect of genotype on progression, adjusted for treatment $(P$ $=0.015 ; \mathrm{OR}=1.84,95 \% \mathrm{CI}=1.12-3.01)$, and a significant effect of treatment on progression, adjusted for genotype $(P=0.001, \mathrm{OR}=1.67,95 \% \mathrm{CI}=1.27-2.19)$ were observed. Combined effect of both the unfavorable LPL-HindIII genotype with moderate drug treatment yielded an odds ratio of 3.30 for progression $(P=0.002,95 \% \mathrm{CI}=$ 1.55-7.03). jects with progression were those with the HindIII 2/2 genotype assigned to the moderate lipid-lowering treatment group (66\%). The lowest percentage of subjects with progression were those with the HindIII $1 / 1$ or $1 / 2$ genotype assigned to the aggressive lipid-lowering treatment group (37\%). MantelHaenszel analysis showed that the effect of genotype on progression, adjusted for treatment, gave an odds ratio of 1.84 (95\%CI 1.13-3.01; $P=0.015)$, and the effect of drug treatment on progression, adjusted for genotype, gave an odds ratio of 1.67 (95\%CI 1.27-2.19; $P=0.001)$. The combined effect of both the unfavorable LPL-HindIII 2/2 genotype and moderate lipid-lowering, when compared with both the favorable genotype and aggressive lipid-lowering, yielded an odds ratio of 3.30 for progression $(95 \% \mathrm{CI}=1.55-7.03, P=0.002)$. This analysis demonstrated that the effect of the LPL-HindIII $2 / 2$ genotype on the progression was independent of, and of the same magnitude as, the effect of the lipid-lowering treatment.

The statistical independence between LPL genotype and drug treatment group was further tested using logistic regression in a model with the dependent variable the presence of progression and with the independent variables LPL-HindIII 2/2 genotype, Lovastatin treatment group, and the interaction between LPL-HindIII genotype and treatment. No significant interaction between LPL-HindIII genotype and drug treatment was observed.

\section{Potential confounding effects}

To investigate the effect of potential confounding variables on the association between LPL-HindIII 2/2 genotype and atherosclerosis progression, the following variables were first tested for association with progression and with genotype independently by univariate analysis: age, ethnicity, gender, current smoking, BMI, history of MI, time between CABG and enrollment in the study, ejection fraction, family history, current medications, systolic and diastolic blood pressure, baseline and steady state lipid levels, and amount of drug needed to reach the target LDL level. Of these, only diastolic blood pressure (DBP) was associated with both LPL-HindIII 2/2 genotype and progression; the mean DBP was $82.1 \mathrm{~mm} \mathrm{Hg}$ for subjects with the LPL-HindIII $2 / 2$ genotype compared with 79.4 $\mathrm{mm} \mathrm{Hg}$ for subjects with other genotypes $(P=0.017, t$ test $)$, and 80.8 for subjects with one or more grafts showing progression compared with 79.1 for subjects without worsening $(P=$ $0.0039, t$ test). However, by applying logistic regression to a model with the presence of atherosclerosis progression as the dependent variable, and with LPL-HindIII 2/2 genotype, DBP, and the interaction between genotype and DBP as independent variables, no significant statistical interaction between genotype and DBP was observed. Lastly, a comparison by logistic regression of two models assessing the effect of LPL-HindIII 2/2 genotype on progression, with or without diastolic blood pressure included in the model, gave similar odds ratios of 1.75 without DBP in the model versus 1.66 with DBP in the model, and similar regression coefficients of 0.56 without DBP versus 0.51 with DBP. When taken together, these results suggested that the effect of the LPL-HindIII 2/2 polymorphism on pro- 
gression is independent of the association of this polymorphism with a higher diastolic blood pressure.

\section{DISCUSSION}

The progression of atherosclerosis in saphenous vein grafts measured in Post-CABG subjects was significantly associated with polymorphisms in the 3 '-section of the $L P L$ gene, $L P L$ $H$ indIII allele 2 , and $L P L-(\text { TTTA })_{n}$ allele 4 . When subjects were stratified by their LPL-HindIII genotype and drug treatment group, both lipid-lowering and LPL genotype had a similar effect on progression of atherosclerosis in the grafts, with odds ratios of 1.8 and 1.7, respectively. This suggests that the magnitude of the LPL genetic effect observed here is similar to the magnitude of the effect of reducing the progression of atherosclerosis using aggressive lipid-lowering therapy.

In contrast, no association between the progression of atherosclerosis in coronary artery bypass grafts and polymorphisms in the 5'-section of the LPL gene, LPL-D9N, N291S, or $P v u I$ I, were observed. This suggests that the as yet unknown functional mutation associated with progression is in linkage disequilibrium with the LPL-(TTTA) $)_{\mathrm{n}}$ and LPL-HindIII polymorphisms, and thus falls in the 3 '-section of the $L P L$ gene.

No associations were observed between the LPL-HindIII polymorphism and baseline or steady state lipid levels, or the amount of drug required to achieve steady state LDL-cholesterol levels. These results suggest that the LPL polymorphism does not act through an effect on the global serum LDL-cholesterol levels. This result is similar to the observed association between the LPL-HindIII 2 allele and the severity of atherosclerosis without concomitant differences in the mean fasting serum lipid levels in a cohort of young myocardial infarction survivors. ${ }^{18}$

The only potential confounding factor that had a significant association with both the LPL-HindIII 2/2 genotype and atherosclerosis progression was diastolic blood pressure (DBP). However, further statistical analysis did not identify an interaction between the genotype and DBP in predicting progression, and furthermore, the LPL-HindIII 2/2 genotype had no greater impact on progression when considered in combination with DBP. This multivariate analysis suggests that a common pathway between DBP, the progression of atherosclerosis in grafts, and the as yet unknown variant(s) in the 3'-section of the LPL gene may exist. How LPL enzymatic activity may impact blood pressure is not immediately obvious; however, we have previously demonstrated linkage of systolic blood pressure with the $L P L$ locus in families with type 2 diabetes from Taiwan..$^{35}$ Because both DBP and atherosclerosis are features of insulin resistance, ${ }^{36-37}$ the as yet unknown $L P L$ variant in the 3 '-section may be acting through its effect on insulin sensitivity. ${ }^{38}$ This is supported by the observations of the association of the LPL gene with components of the insulin resistance syndrome in both human and mouse models. ${ }^{15,21,38-40}$ Alternatively, LPL increases expression of nitric oxide synthase in macrophages in vitro, ${ }^{41}$ and such an effect in vessel wall mac- rophages may influence blood pressure. In this same system, glucose has been shown to upregulate $L P L$ expression as well. ${ }^{42}$

A potential limitation of this study is the observation of significant differences in important atherosclerosis risk factors between subjects included in the study and those not included. Genetic study subjects had fewer clinical events and were perhaps more compliant with greater frequency of aspirin use, less tobacco use, and more favorable stead-state LDL and HDL values. This bias likely occurred because patients for the genetic study were collected in the latter years of the clinical trial. However, given the direction of the data reported here, it is also likely that, as the result of this bias we have underestimated the strength of the LPL-HindIII $2 / 2$ association with progression because our cohort is composed of subjects with improved risk factors for survival.

In conclusion, we report an association between the $L P L$ HindIII 2/2 genotype and the quantitative progression of atherosclerosis in grafts measured over 4 years in the Post-CABG Trial. The known haplotype structure of the $L P L$ gene suggests that variation in the 3 '-section of the $L P L$ gene, in linkage disequilibrium with the LPL-HindIII 2 allele, is a risk factor for the progression of atherosclerosis that is independent and additive to the aggressive use of lovastatin to reduce LDL-cholesterol. Its effect on progression was of the same magnitude as the use of moderate rather than aggressive lipid lowering treatment.

\section{ACKNOWLEDGMENTS}

This work was supported in part by grants from the Parsons Foundation, the Steven S. Cohen Heart Fund, the Abe and Estelle and Marjorie Sanders Foundation, and the Cedars-Sinai Board of Governors' Chair in Medical Genetics. Maintenance of the EBV-transformed cell lines is supported by the National Center for Research Resources, grant M01-RR00425. The Post-CABG Trial was supported by research contracts with the National Heart, Lung, and Blood Institute and by Merck \& Co. Lovastatin was donated by Merck \& Co.; warfarin and placebo were donated by Dupont Pharma; cholestyramine and placebo were donated by Bristol-Myers Squibb; aspirin was donated by Bayer; modified Biotrack machines were provided by Biotrack. We thank Darlene Goldstein and Zhiming Li for assistance with statistical analyses performed during preliminary stages of this work.

\section{References}

1. Post-CABG Trial Investigators. The effect of aggressive lowering of low-density lipoprotein cholesterol levels and low-dose anticoagulation on obstructive changes in saphenous-vein coronary-artery bypass grafts. N Engl J Med 1997;336:153-162.

2. Lusis AJ. Atherosclerosis. Nature 2000;407:233-24.

3. Benlian P, De Gennes JL, Foubert L, Zhang H, Gagne SE, Hayden M. Premature atherosclerosis in patients with familial chylomicronemia caused by mutations in the lipoprotein lipase gene. N Engl J Med 1996;335:848-854.

4. Mailly F, Tugrul Y, Reymer PW, Bruin T, Seed M, Groenemeyer BF et al. A common variant in the gene for lipoprotein lipase (Asp9->Asn). Functional implications and prevalence in normal and hyperlipidemic subjects. Arterioscler Thromb Vasc Biol 1995; 15:468-478.

5. Wittrup HH, Tybjaerg-Hansen A, Steffensen R, Deeb SS, Brunzell JD, Jensen G et al. Mutations in the lipoprotein lipase gene associated with ischemic heart disease in men. The Copenhagen city heart study. Arterioscler Thromb Vasc Biol 1999;19:15351540 . 


\section{Taylor et al.}

6. Kastelein JJ, Groenemeyer BE, Hallman DM, Henderson H, Reymer PW, Gagne SE et al. The Asn9 variant of lipoprotein lipase is associated with the -93G promoter mutation and an increased risk of coronary artery disease. The Regress Study Group. Clin Genet 1998;53:27-33.

7. Ruel IL, Gaudet D, Perron P, Bergeron J, Julien P, Lamarche B. Characterization of LDL particle size among carriers of a defective or a null mutation in the lipoprotein lipase gene: the Quebec LIPD Study. Arterioscler Thromb Vasc Biol 2002;22:11811186.

8. Jukema JW, van Boven AJ, Groenemeijer B, Zwinderman AH, Reiber JH, Bruschke AV et al. The Asp9 Asn mutation in the lipoprotein lipase gene is associated with increased progression of coronary atherosclerosis. REGRESS Study Group, Interuniversity Cardiology Institute, Utrecht, The Netherlands. Regression Growth Evaluation Statin Study. Circulation 1996;94:1913-1918.

9. Reymer PW, Gagne E, Groenemeyer BE, Zhang H, Forsyth I, Jansen H et al. A lipoprotein lipase mutation (Asn291Ser) is associated with reduced HDL cholesterol levels in premature atherosclerosis. Nat Genet 1995;10:28-34.

10. Wittrup HH, Tybjaerg-Hansen A, Nordestgaard BG. Lipoprotein lipase mutations plasma lipids and lipoproteins, and risk of ischemic heart disease. A meta-analysis. Circulation 1999;99:2901-2907.

11. Pimstone SN, Clee SM, Gagne SE, Miao L, Zhang H, Stein EA et al. A frequently occurring mutation in the lipoprotein lipase gene (Asn291Ser) results in altered postprandial chylomicron triglyceride and retinyl palmitate response in normolipidemic carriers. J Lipid Res 1996;37:1675-1684.

12. Gerdes C, Fisher RM, Nicaud V, Boer J, Humphries SE, Talmud PJ et al. Lipoprotein lipase variants D9N and N291S are associated with increased plasma triglyceride and lower high-density lipoprotein cholesterol concentrations: studies in the fasting and postprandial states: the European Atherosclerosis Research Studies. Circulation 1997;96:733-740.

13. Mero N, Suurinkeroinen L, Syvanne M, Knudsen P, Yki-Jarvinen H, Taskinen MR Delayed clearance of postprandial large TG-rich particles in normolipidemic carriers of LPL Asn291Ser gene variant. J Lipid Res 1999;40:1663-1670.

14. Wittrup HH, Tybjaerg-Hansen A, Abildgaard S, Steffensen R, Schnohr P, Nordestgaard BG. A common substitution (Asn291Ser) in lipoprotein lipase is associated with increased risk of ischemic heart disease. J Clin Invest 1997;99:1606-1613.

15. Klannemark M, Suurinkeroinen L, Orho-Melander M, Groop L, Taskinen MR. Interaction between the Asn291Ser variant of the LPL gene and insulin resistance on dyslipidaemia in high risk individuals for Type 2 diabetes mellitus. Diabet Med 2000;17:599-605.

16. Wang XL, McCredie RM, Wilcken DE. Common DNA polymorphisms at the lipoprotein lipase gene. Association with severity of coronary artery disease and diabetes. Circulation 1996;93:1339-1345.

17. Mattu RK, Needham EW, Morgan R, Rees A, Hackshaw AK, Stocks J et al. DNA variants at the LPL gene locus associate with angiographically defined severity of atherosclerosis and serum lipoprotein levels in a Welsh population. Arterioscler Thromb 1994;14:1090-1097.

18. Peacock RE, Hamsten A, Nilsson-Ehle P, Humphries SE. Associations between lipoprotein lipase gene polymorphisms and plasma correlations of lipids, lipoproteins and lipase activities in young myocardial infarction survivors and age-matched healthy individuals from Sweden. Atherosclerosis 1992;97:171-185.

19. Chen L, Patsch W, Boerwinkle E. HindIII DNA polymorphism in the lipoprotein lipase gene and plasma lipid phenotypes and carotid artery atherosclerosis. Hum Genet 1996;98:551-556.

20. Anderson JL, King GJ, Bair TL, Elmer SP, Muhlestein JB, Habashi J et al. Association of lipoprotein lipase gene polymorphisms with coronary artery disease. J Am Coll Cardiol 1999;33:1013-1020.
21. Ahn YI, Ferrell RE, Hamman RF, Kamboh MI. Association of lipoprotein lipase gene variation with the physiological components of the insulin-resistance syndrome in the population of the San Luis Valley, Colorado. Diabetes Care 1993;16:1502-1506.

22. Nickerson DA, Taylor SL, Weiss KM, Clark AG, Hutchinson RG, Stengard J et al DNA sequence diversity in a 9.7-kb region of the human lipoprotein lipase gene. Nat Genet 1998;19:233-240.

23. Clark AG, Weiss KM, Nickerson DA, Taylor SL, Buchanan A, Stengard J et al. Haplotype structure and population genetic inferences from nucleotide-sequence variation in human lipoprotein lipase. Am J Hum Genet 1998;63:595-612.

24. Templeton AR, Clark AG, Weiss KM, Nickerson DA, Boerwinkle E, Sing CF. Recombinational and mutational hotspots within the human lipoprotein lipase gene. Am J Hum Genet 2000;66:69-83.

25. Templeton AR, Weiss KM, Nickerson DA, Boerwinkle E, Sing CF. Cladistic structure within the human Lipoprotein lipase gene and its implications for phenotypic association studies. Genetics 2000;156:1259-1275.

26. Anderson MA, Gusella JF. Use of cyclosporin A in establishing Epstein-Barr virustransformed human lymphoblastoid cell lines. In Vitro 1984;20:856-858.

27. Miller G, Lipman M. Release of infectious Epstein-Barr virus by transformed marmoset leukocytes. Proc Natl Acad Sci U S A 1973;70:190-194.

28. Freshney R. Culture of animal cells: A manual of basic techniques. New York: Liss, 1987.

29. Pressman S, Rotter JI. Epstein-Barr virus transformation of cryopreserved lymphocytes: prolonged experience with technique. Am J Hum Genet 1991;49:467.

30. Sambrook J, Fritsch E, Maniatis T. Molecular cloning. New York: Cold Spring Harbor Laboratory, 1989.

31. Li SR, Oka K, Galton D, Stocks J. Pvu-II RFLP at the human lipoprotein lipase (LPL) gene locus. Nucleic Acids Res 1988;16:2358.

32. Zuliani G, Hobbs HH. Tetranucleotide repeat polymorphism in the LPL gene. $N u$ cleic Acids Res 1990;18:4958.

33. Heizmann C, Kirchgessner T, Kwiterovich PO, Ladias JA, Derby C, Antonarakis SE et al. DNA polymorphism haplotypes of the human lipoprotein lipase gene: possible association with high density lipoprotein levels. Hum Genet 1991;86:578-584.

34. SAS Institute [computer program]. Release 6.12. Cary, NC: SAS Institute, 1997.

35. Wu DA, Bu X, Warden CH, Shen DD, Jeng CY, Sheu WH et al. Quantitative trait locus mapping of human blood pressure to a genetic region at or near the lipoprotein lipase gene locus on chromosome 8p22. J Clin Invest 1996;97:2111-2118.35.

36. Reaven GM. Banting lecture 1988. Role of insulin resistance in human disease Diabetes 1988;37:1595-1607.

37. Bloomgarden ZT. Genes and the cell biology of insulin secretion and insulin resistance. Diabetes Care 1999;22:849-853.

38. Goodarzi MO, Guo X, Taylor KD, Quinones MJ, Saad MF, Yang H et al. Lipoprotein lipase is a gene for insulin resistance in Mexican Americans. Diabetes 2004;53:214220

39. Xiang AH, Azen SP, Buchanan TA, Raffel LJ, Tan S, Cheng LS et al. Heritability of subclinical atherosclerosis in Latino families ascertained through a hypertensive parent. Arterioscler Thromb Vasc Biol 2002;22:843-848.

40. Ferreira LD, Pulawa LK, Jensen DR, Eckel RH. Overexpressing human lipoprotein lipase in mouse skeletal muscle is associated with insulin resistance. Diabetes 2001 50:1064-1068.

41. Renier G, Lambert A. Lipoprotein lipase synergizes with interferon gamma to in duce macrophage nitric oxide synthetase mRNA expression and nitric oxide production. Arterioscler Thromb Vasc Biol 1995;15:392-399.

42. Sartippour MR, Lambert A, Laframboise M, St-Jacques P, Renier G. Stimulatory effect of glucose on macrophage lipoprotein lipase expression and production. $\mathrm{Di}$ abetes $1998 ; 47: 431-438$. 